\title{
Proximal tibiofibular synostosis as a possible cause of a pseudoradicular syndrome: a case report
}

\author{
Bas van Ooij · André van Ooij · J. Wim Morrenhof • \\ C. Niek van Dijk
}

Received: 17 August 2010/Accepted: 16 December 2010/Published online: 11 January 2011

(C) The Author(s) 2011. This article is published with open access at Springerlink.com

\begin{abstract}
This paper presents a case report of persistent low back pain and suspected lumbar radiculopathy. A synostosis at the level of the proximal tibiofibular joint was diagnosed. After successful resection of the synostosis, the low back symptoms resolved completely. This is the first report of a proximal tibiofibular synostosis as a possible cause of referred pain proximally.
\end{abstract}

Keywords Proximal tibiofibular joint - Synostosis . Pseudoradicular syndrome

\section{Introduction}

Disorders at the level of the proximal tibiofibular joint are rare. The most common neoplastic disorders are the proximal tibiofibular synostosis $[1,2,5,7,8,10-13,17$, 20], the fibular head osteochondroma and an exostosis with possible peroneal paralysis, often associated with multiple hereditary osteochondromas [3, 4, 6, 9, 14, 16, 18]. Besides the possible peroneal paralysis, lateral knee and ankle pain are the most common symptoms in patients with one of these disorders $[2,8,10,16,17]$. To our knowledge, an association with low back pain or a pseudoradicular syndrome has not yet been described. A case report of a patient

B. van Ooij $(\square) \cdot$ C. N. van Dijk

Department of Orthopaedic Surgery, Academic Medical Centre and the Orthopaedic Research Centre Amsterdam,

Room G4-242, Meibergdreef 9, 1105 AZ Amsterdam,

The Netherlands

e-mail: B.vanOoij@amc.uva.nl

A. van Ooij · J. W. Morrenhof

Department of Orthopaedic Surgery,

VieCuri Medical Centre, Venlo, The Netherlands with persistent low back pain and suspected lumbar radiculopathy is presented in which a proximal tibiofibular synostosis was diagnosed.

\section{Case report}

A 49-year-old man presented with a 4-year history of low back pain and referred pain in his right leg. While standing, he felt sensory sensations from the lateral side of his right upper leg to the knee and the medial side of his lower leg and ankle. Coughing and sneezing worsened these symptoms. He complained about some weakness of his leg, but he had no pain or other symptoms of his knee. Apart from a trochanteric bursitis 5 years earlier, his medical record reported no relevant details. Physical examination showed a restricted flexion of his lumbar spine, with pain at the level of the right sacroiliac joint during lateroflexion and extension of the lumbar spine. Local tenderness was noticed at the level of the right sacroiliac joint, gluteal and trochanteric region. Neurologic examination did not reveal any abnormalities: no weakness was noticed in his right leg, and the straight leg and femoral stretch tests were negative.

Plain radiographs of the SI-joint, pelvis and hip did not reveal any abnormality. Also an MRI of the lumbar vertebrae revealed no abnormality, especially no herniated disc. Laboratory data showed no abnormal blood levels. A technetium scintigraphic scan showed no increased isotope uptake in the lower spine, but revealed a hot spot at the level of the proximal tibiofibular joint (Fig. 1).

At a second physical examination, local tenderness at the level of the proximal tibiofibular joint was present. There was a normal mechanical axis of the right leg and the knee function was optimal. Radiographs of the right knee showed proliferative bone formation between the proximal tibia and 
Fig. 1 Technetium scintigraphic scan. No increased isotope uptake of the lower spine, but a hotspot at the level of the right proximal tibiofibular joint is shown

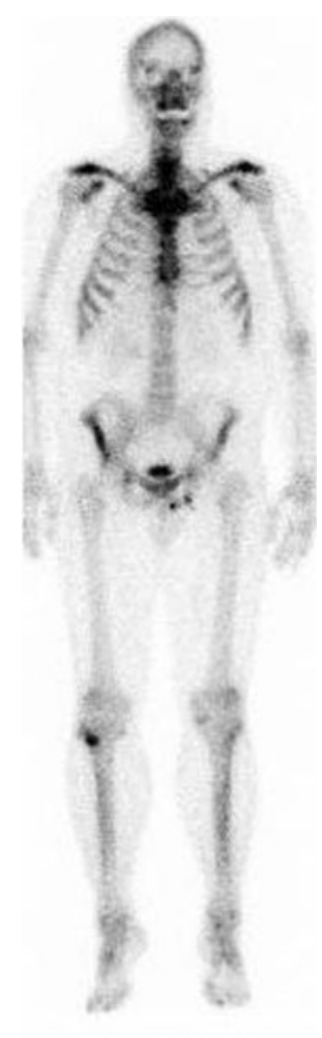

RE ANTERIOR U

fibula. There was no dislocation or subluxation of the proximal tibiofibular joint, and the proximal fibula had a normal shaft without any bowing. A CT scan showed eccentric bone growth of both the proximal tibia and fibula, causing a synostosis at the edges of the bone formations (Fig. 2).

An excision of the synostosis was performed with an additional peroneal nerve release. At surgery, no cartilage cap was found, indicating eccentric bone growth rather than an osteochondroma. The synostosis was removed until the fibula could move entirely free from the tibia. The peroneal nerve was not compressed by the synostosis, and it had a normal appearance.

Postoperatively, the pain at the level of the tibiofibular joint decreased, and surprisingly, the low back pain

symptoms and referred pain disappeared entirely. After 2 months, he started giving soccer training again, which was impossible preoperatively. Postoperative radiographs and CT scan revealed a little remnant of the tibial bone growth, but the synostosis appeared to have been removed completely. After 7 months, he complained of pain in the fibular head again, with referred pain to the ankle. He did not complain about low back pain or other related symptoms. At the last follow-up examination at 10 months postoperatively, he was free of symptoms.

\section{Discussion}

Reports of proximal tibiofibular synostosis are rare $[1,2,5$, 7, 8, 10-13, 17, 20]. Rahm was the first (in 1924) to describe a case of a synostosis in a 43-year-old patient [13]. Most of the reported proximal tibiofibular synostosis developed before closure of the growth plates or congenitally $[1,5,11,12,20]$. Osteochondroma leading to synostosis has been reported once [2]. Takai et al. [17] reported a synostosis after closure of the growth plate in a 24-year-old volleyball player. They referred to the classification of O'Dwyer, published in 1991 (Fig. 3) [12].

Takai et al. proposed that the classification of O'Dwyer [12] should be extended with a type-4 synostosis [17], because no deformity, bowing or length discrepancy of the fibula was present in their case. They suggested that the type-4 synostosis occurred after closure of the growth plate, with repeated mechanical stress to the tibiofibular joint as a possible cause. Because no deformity, bowing or length discrepancy of the fibula was present in our patient, we can confirm that adding a type 4 to the classification of O'Dwyer [12] would be logical. At revision of the literature, the presented patient by Nishikawa et al. [10] had another type-4 synostosis.

A pseudoradicular syndrome in combination with a fibular head abnormality, as a result of non-specific fibrosis, with peroneal nerve entrapment was described by Saal et al. [15]. They suggested that a lower extremity nerve
Fig. 2 Coronal (a) and transverse (b) CT scan of the right knee. The proliferative bone formation causes a proximal tibiofibular synostosis
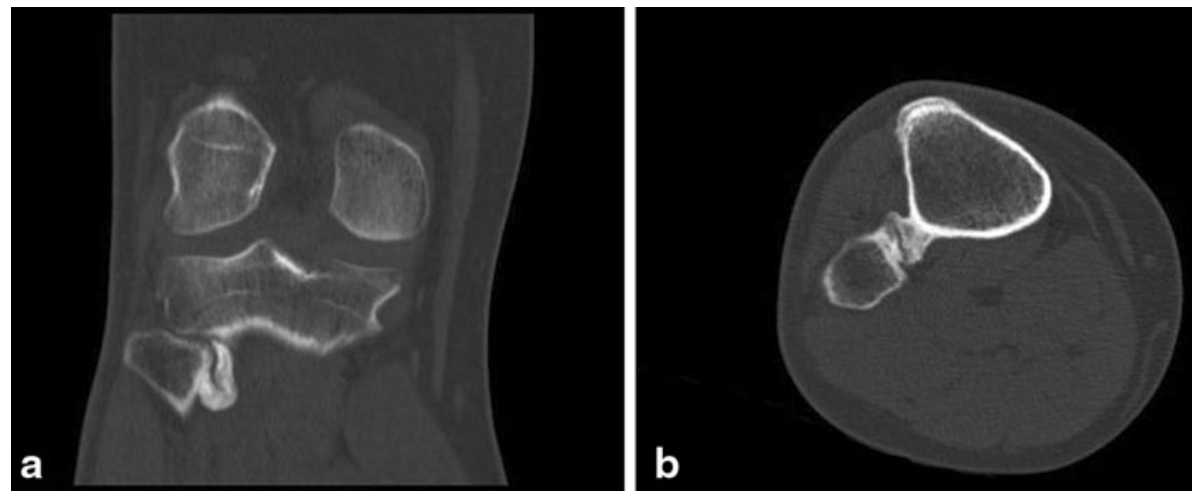


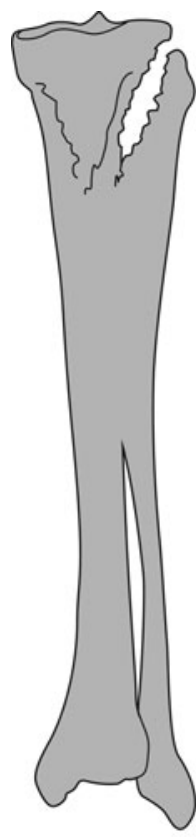

I

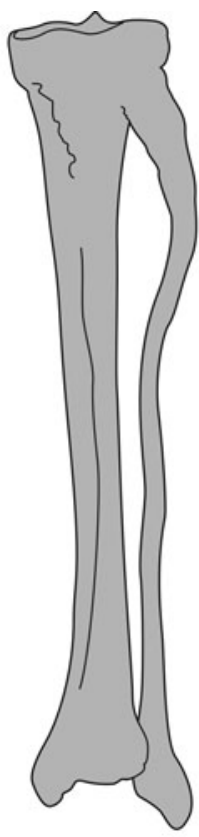

II

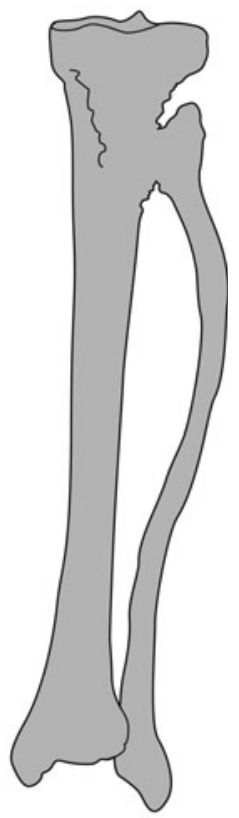

III
Fig. 3 Classification of proximal tibiofibular synostosis as published by O'Dwyer [12] (adapted with permission). Type 1 represents a straight fibula with a large synostosis arising from the proximal to the middle and distal thirds of the tibia and fibula [20], assumed to be caused by a trauma. Type 2 represents a synostosis at the level of the proximal tibiofibular joint with a normal fibular length and a mild bowing in the proximal fibula; this type was reported in 3 cases $[5,12,20]$. Type 3 represents a more distal synostosis than type 2 with a marked bowing of the fibula (and widening of the interosseous distance) throughout its length; this type was reported in 3 cases $[1,7,13]$

entrapment could mimic a lumbar pain syndrome. Of 45 patients with peripheral nerve entrapment (femoral, saphenous, peroneal or tibial nerve), $49 \%$ had low back complaints. Twenty peroneal lesions were noted at or above the fibular head, of which six patients (30\%) were presented with low back pain.

Although we did not find a peroneal entrapment, our patient had a painful and abnormal spinal range of motion, indicating a masquerading lumbar radiculopathy as described by Saal et al. [15]. They performed surgical neurolysis in 12 cases (60\%); non-specific fibrosis at and above the fibular head was present in all cases. They referred to the theory that neural activation of the dorsal root ganglion could cause referred pain in the same pattern as in lumbar pain syndromes [19]. Although we did not notice fibrosis at or above the fibular head in our patient, theoretically the synostosis could have caused stretching of the peroneal nerve, causing dorsal root ganglion activation. Speculative, the coughing and sneezing which worsened the leg symptoms in our patient could have stimulated this activation. Because no herniated disc was present, this would have been referred stimulation of the sciatic nerve.
Another possible cause of low back pain in their patients could be the mechanical factors created by asymmetrical gait patterns and knee instability (noted in 9 cases) [15]. These subtle alterations in gait mechanics could be another plausible explanation in our patient although he had a symmetrical gait pattern and no knee instability.

\section{Conclusion}

This is the third report of a type- 4 proximal tibiofibular synostosis and the first report of a tibiofibular synostosis as a possible cause of referred pain proximally. It can be concluded that when the source of (pseudo)radicular pain is not very obvious, a peripheral source has to be considered, even if no peripheral symptoms are present at physical examination. To differentiate between a lumbar or possible peripheral source, a technetium scintigraphic scan could be a valuable additional test.

Open Access This article is distributed under the terms of the Creative Commons Attribution Noncommercial License which permits any noncommercial use, distribution, and reproduction in any medium, provided the original author(s) and source are credited.

\section{References}

1. Bergmann E (1941) Congenital tibiofibular synostosis. Int Coll Surg 4:359-360

2. Bozkurt M, Dogan M, Turanli S (2004) Osteochondroma leading to proximal tibiofibular synostosis as a cause of persistent ankle pain and lateral knee pain: a case report. Knee Surg Sports Traumatol Arthrosc 12:152-154

3. Cardelia JM, Dormans JP, Drummond DS, Davidson RS, Duhaime C, Sutton L (1995) Proximal fibular osteochondroma with associated peroneal nerve palsy: a review of six cases. J Pediatr Orthop 15:574-577

4. Flores LP, Koerbel A, Tatagiba M (2005) Peroneal nerve compression resulting from fibular head osteophyte-like lesions. Surg Neurol 64:249-252

5. Gamble JG (1984) Proximal tibiofibular synostosis. J Pediatr Orthop 4:243-245

6. Hazmy W (2001) Peroneal nerve palsy secondary to proximal fibular osteochondroma - a case report. Med J Malaysia 56(Suppl C):89-90

7. Hippe H (1953) A rare proximal tibia-fibular synostosis. Fortschr Geb Rontgenstr 78:748-749

8. Lenin BV, Shenbaga N, Komarasamy B, Paul A (2006) Proximal tibiofibular synostosis as a source of ankle pain: a case report. Iowa Orthop J 26:127-129

9. Mnif H, Koubaa M, Zrig M, Zammel N, Abid A (2009) Peroneal nerve palsy resulting from fibular head osteochondroma. Orthopedics $32: 528$

10. Nishikawa T, Yamamoto T, Yoshiya S, Kurosaka M (2003) Intermittent peroneal mononeuropathy due to proximal tibiofibular synostosis. J Neurol 250:352-353

11. Nishimura T, Nii E, Urawa M, Nishiyama M, Taki S, Uchida A (2008) Proximal tibiofibular synostosis with 49, XXXXY syndrome, a rare congenital bone anomaly. J Orthop Sci 13:390-395 
12. O’Dwyer KJ (1991) Proximal tibio-fibular synostosis. A rare congenital anomaly. Acta Orthop Belg 57:204-208

13. Rahm H (1924) Die Tibiofibulare Synostose. Zeitschrift Orthop Chir 43:64-69

14. Rinaldi E (1983) Peroneal paralysis due to exostosis of the fibula. Report of 2 cases. Ital J Orthop Traumatol 9:259-262

15. Saal JA, Dillingham MF, Gamburd RS, Fanton GS (1988) The pseudoradicular syndrome. Lower extremity peripheral nerve entrapment masquerading as lumbar radiculopathy. Spine (Phila Pa 1976) 13:926-930

16. Takai S, Yoshino N, Aso S, Hirasawa Y (2001) Symptomatic spur formation of bilateral proximal tibiofibular joints. Orthopedics 24:843-845
17. Takai S, Yoshino N, Hirasawa Y (1999) Unusual proximal tibiofibular synostosis. Int Orthop 23:363-365

18. Watson LW, Torch MA (1993) Peroneal nerve palsy secondary to compression from an osteochondroma. Orthopedics 16:707-710

19. Weinstein J (1986) Report of the 1985 ISSLS Traveling Fellowship. Mechanisms of spinal pain. The dorsal root ganglion and its role as a mediator of low-back pain. Spine (Phila Pa 1976) 11:999-1001

20. Wong K, Weiner DS (1978) Proximal tibiofibular synostosis. Clin Orthop Relat Res 135:45-47 